\title{
A Kalman filtering algorithm for regularization networks
}

\author{
G. De Nicolao, G. Ferrari-Trecate \\ Dipartimento di Informatica e Sistemistica, Università di Pavia, \\ Via Ferrata 1, 27100 Pavia, Italy. \\ Phone: +39.382 .505484 Fax: +39.382 .505373$ \\ E-mail: \{denicolao, ferrari\}oconpro.unipv.it
}

\begin{abstract}
Regularization networks are nonparametric estimators obtained from the application of Tychonov regularization or Bayes estimation to the hypersurface reconstruction problem. With the usual algorithm, the computation of the weights scales as $O\left(n^{3}\right)$ where $n$ is the number of data. In this paper we show that for a class of monodimensional problems, the complexity can be reduced to $O(n)$ by a suitable algorithm based on spectral factorization and Kalman filtering. The procedure applies also to smoothing splines and, in a multidimensional context, to additive regularization networks.
\end{abstract}

\section{Introduction}

At the beginning of the nineties Poggio and Girosi [12] made a notable attempt at giving theoretical grounds to the use of neural networks in terms of approximation theory. Approaching the hypersurface reconstruction problem by means of regularization theory, they showed that under suitable assumptions the solution is an RBF-like (RBF: Radial Basis Function) network that they called Regularization Network (RN). For RN the number of neurons equals the number $n$ of datapoints and the activation functions are a set of Green's functions whose characterization depends on the form of the stabilizer appearing in the regularization cost functional [12].

The same result, but with a different route, is obtained by formulating the hypersurface reconstruction problem within the framework of Bayes estimation. Indeed, the a-priori knowledge on the hypersurface can be represented by assuming that the unknown function is a Gaussian random field having an assigned autocovariance function [18]. Then, if the measurement errors are Gaussian as well, the Bayes estimate is again a RN [17].

Besides their nice theoretical interpretation, RNs enjoy a remarkable consistency property, in the sense that, under mild assumptions, their estimate converges to the true hypersurface as the number of training data tends to infinity [3], [4].

In spite of their twofold theoretical justification, the use of RNs is not so broad as expected because the training of RNs appears to be computationally expensive $\left(O\left(n^{3}\right)\right.$ operations). In order to overcome this " $n^{3}$ curse", simpler suboptimal approximations were proposed such as Generalized RBF's and Hyper Basis Functions networks $[12,7]$.

However, there is a notable class of RNs for which the $O\left(n^{3}\right)$ curse has been overcome. In the statistical literature, it is known that the so-called ARMA splines can be computed with $O(n)$ complexity by applying Kalman filtering to a suitable state-space model [16], [2]. These $O(n)$ algorithms, however, are oriented towards the pointwise computation of the regression function over a prespecified grid rather than providing directly the weights of the RN. Hence, the network structure of the estimator is lost together with its possible advantages in terms of parallel implementation.

The main purpose of the present paper is to show that in some specific, yet significant case the RN weights can be computed with $O(n)$ complexity. Our result concerns a fairly large class of RNs for the one-dimensional problem where the function to be reconstructed is a scalar map of a scalar variable. Although this is a limitation, there are significant signal reconstruction problems involving very large data sets which can benefit substantially from the introduction of an $O(n)$ algorithm, see e.g. the anaesthesiology problem studied in [5], where the data for a single subject involve roughly $50,000 / 100,000$ observations collected at a nonuniform sampling rate. The derivation of the $O(n)$ algorithm is based on the Bayesian interpretation of RNs, and, more precisely on a key technical lemma showing that the covariance matrix of an $n$-dimensional random vector obtained by sampling $n$ times a Markovian stochastic process can be inverted in $O(n)$ operations by Kalman filtering. As a particular case, the algorithm can be applied also to smoothing splines that can be regarded as a particular type of RNs.

It is unfortunate that the monodimensional algorithm cannot be extended to generic multidimensional RNs. 
Nevertheless, an important exception is given by additive RNs which represent a function of several variables as the sum of scalar functions [7].

\section{Preliminaries}

Consider the regression problem of estimating an unknown function $f(\cdot): \mathbb{R}^{d} \rightarrow \mathbb{R}$, from the noisy observations

$$
y_{i}=f\left(t_{i}\right)+\epsilon_{i}, \quad i=1, \ldots, n
$$

where the additive measurement errors $\epsilon_{i}$ are uncorrelated zero-mean Gaussian random variables with $\operatorname{Var}\left[\epsilon_{i}\right]=\sigma^{2}$. The training set will be denoted by $\mathcal{D}_{n}=$ $\{\bar{t}, \bar{y}\}$, where $\bar{t}=\left[t_{1}, \ldots, t_{n}\right]^{\prime}$ and $\bar{y}=\left[y_{1}, \ldots, y_{n}\right]^{\prime}$.

Regularization networks [7] constitute a theoretically grounded nonparametric method for solving such regression problems.

Definition 1 A map $C\left(\xi_{1}, \xi_{2}\right): \mathbb{R}^{d} \times \mathbb{R}^{d} \rightarrow \mathbb{R}$ is positive-definite if it is symmetric (i.e. $C\left(\xi_{1}, \xi_{2}\right)=$ $\left.C\left(\xi_{2}, \xi_{1}\right)\right)$ and satisfies, $\forall n \in \mathbb{N}_{0}$

$\sum_{i=1}^{n} \sum_{j=1}^{n} a_{i} a_{j} C\left(t_{i}, t_{j}\right) \geq 0, \forall a_{i} \in \mathbb{R}, \forall a_{j} \in \mathbb{R}, \forall t_{i}, t_{j} \in \mathbb{R}^{d}$

Definition 2 Given the training set $\mathcal{D}_{n}$, a positivedefinite continuous function $C\left(\xi_{1}, \xi_{2}\right): \mathbb{R}^{d} \times \mathbb{R}^{d} \rightarrow \mathbb{R}$ and a scalar $\gamma \geq 0$, the associated regularization network $(\mathrm{RN})$ is the mapping $f_{\gamma n}(t): \mathbb{R}^{d} \rightarrow \mathbb{R}$

$$
f_{\gamma n}(t)=\sum_{k=1}^{n} \theta_{k} C\left(t, t_{k}\right)
$$

where

$$
\begin{gathered}
\bar{\theta} \doteq\left[\begin{array}{cc}
\theta_{1}, & \ldots, \theta_{n}
\end{array}\right]^{\prime}=(H+\gamma I)^{-1} \bar{y} \\
{[H]_{i j} \doteq C\left(t_{i}, t_{j}\right) .}
\end{gathered}
$$

Remark 1 If $C\left(\xi_{1}, \xi_{2}\right)$ is radial, i.e. $C\left(\xi_{1}, \xi_{2}\right)=$ $h\left(\| \xi_{1}-\xi_{2}||\right)$, then it can be shown [6] that $f_{\gamma n}(\cdot)$ is the solution of the Tychonov-like regularization problem

$$
f_{\gamma n}(t)=\min _{f} \sum_{k=1}^{n}\left(y_{k}-f\left(t_{k}\right)\right)^{2}+\gamma \int_{\mathbb{R}^{d}} \frac{|\mathcal{F}(f)(\omega)|^{2}}{\mathcal{F}(h)(\omega)} d \omega,
$$

where $\mathcal{F}$ denotes the (d-dimensional) Fourier transform operator. Moreover it turns out that the RN is just a Radial Basis Function neural network whose coefficients can be computed from (3).

A major drawback of RNs is that, in general, the exact calculation of the vector $\bar{\theta}$ scales as $O\left(n^{3}\right)$ due to the computational complexity of the linear system $(H+$ $\gamma I) \bar{\theta}=\bar{y}$. In order to derive an $O(n)$ algorithm for the calculation of these coefficients, it is first convenient to introduce the Bayesian interpretation of Regularization Networks.

In Bayes estimation a statistical distribution (the socalled prior) for the unknown function $f(\cdot)$ must be assigned. In particular, we model $f(\cdot)$ as a zero-mean $d$-dimensional Gaussian (stochastic) process (independent of the errors $\epsilon_{i}$ ) with autocovariance function

$$
\mathrm{E}\left[f\left(\xi_{1}\right) f\left(\xi_{2}\right)\right]=C\left(\xi_{1}, \xi_{2}\right) .
$$

Then, it turns out that [17]

$$
\begin{aligned}
\hat{f}(t) & \doteq \mathrm{E}\left[f \mid \mathcal{D}_{n}\right](t)=\sum_{k=1}^{n} \theta_{k} C\left(t, t_{k}\right) \\
\mathrm{E}\left[\bar{y} \bar{y}^{\prime}\right] \bar{\theta} & =\bar{y}, \quad \mathrm{E}\left[\bar{y} \bar{y}^{\prime}\right]=\left(H+\sigma^{2} I\right) .
\end{aligned}
$$

It is apparent that $\hat{f}(t)=f_{\gamma n}(t)$ if $\gamma=\sigma^{2}$. The map $\hat{f}(t)$ is the Bayes estimate.

By the way, note that there exists a one-to one correspondence between positive definite functions and autocovariance functions of Gaussian processes [11].

Hereafter, we will restrict our attention to monodimensional regression problems $(d=1)$, In other words, we are concerned with the problem of reconstructing a function $f(\cdot): \mathbb{R} \rightarrow \mathbb{R}$ given $n$ noisy samples $y_{i}$, see (1). In this case, we assume that the "time samples" $\left\{t_{i}\right\}$ are ordered, i.e. $t_{1}<t_{2}<\ldots<t_{n}$. The interpretation of the input $t$ as a time variable opens the way to the use of Kalman filtering techniques, at least when the autocovariance $C\left(\xi_{1}, \xi_{2}\right)$ admits a state-space representation. In particular, in the present and the following Section, we will consider stationary autocovariance functions $h(\cdot)$ satisfying the following assumption.

Assumption A1 Letting $H(s)$ be the bilateral Laplace transform of $h(\cdot)$, assume that

a. $H(0)>0$

b. $H(s)$ is a rational function of the type

$$
\begin{aligned}
& H(s)=s^{2 m^{\prime}} \frac{N_{H}(s)}{D_{H}(s)}= \\
& =s^{2 m^{\prime}} \frac{a_{0} s^{2 m}+a_{1} s^{2(m-1)}+\ldots+a_{m}}{s^{2 p}+b_{1} s^{2(p-1)}+\ldots+b_{p}}
\end{aligned}
$$

where $a_{0}, a_{1}, \ldots, a_{m}, b_{1}, b_{2}, \ldots, b_{p}$ are real coeffcients and $m, m^{\prime}, p$ are suitable nonnegative integers satisfying $m+m^{\prime}<p, p>0$.

c. No root of $D_{H}(s)$ and $N_{H}(s)$ lies on the imaginary axis. 
Assumption A1 is not very restrictive even though it rules out autocovariance functions whose Laplace transform $H(s)$ is not the ratio of polynomials in the $s^{2}$ variable. For instance, the squared-exponential autocovariance function $h\left(\left|\xi_{1}-\xi_{2}\right|\right)=\exp \left(-\left|\xi_{1}-\xi_{2}\right|^{2}\right)$ is not allowed, since $H(s)=\sqrt{\pi} \exp \left(s^{2} / 4\right)$. However, in view of Runge's theorem [13] one may approximate such (holomorphic) $H(s)$ with arbitrary precision on compact subsets of the complex plane by using rational functions.

\section{Derivation of the fast algorithm}

The advantage of using autocovariance functions satisfying Assumption A1 is that the corresponding Gaussian process $f(\cdot)$ can be represented as the output $\tilde{f}(t)$ of a linear time-invariant system admitting a statespace representation of the type:

$$
\mathcal{T}:\left\{\begin{array}{rl}
d x(t) & =A x(t) d t+B d w(t) \\
\tilde{f}(t) & =C x(t) \\
x\left(t_{0}\right) & =x_{0}
\end{array},\right.
$$

where $A \in \mathbb{R}^{p \times p}, B \in \mathbb{R}^{p \times 1}, C \in \mathbb{R}^{1 \times p}$ are suitable matrices, $w(\cdot)$ is a Wiener process, and the initial state $x_{0}$ is a suitable random vector. The next three Lemmas show how to obtain the state-space representation (6) from the spectrum $H(s)$ as well as how to derive the corresponding discrete time model at the sampling points. Due to the lack of space, proofs are omitted.

Lemma 1 Let Assumption A1 holds. Then there exists a complex function

$$
G(s) \doteq \frac{N_{G}(s)}{D_{G}(s)}=\frac{\tilde{a}_{1} s^{p-1}+\tilde{a}_{2} s^{p-2}+\ldots+\tilde{a}_{p}}{s^{p}+\tilde{b}_{1} s^{p-1}+\ldots+\tilde{b}_{p}}
$$

where $\tilde{a}_{1}, \tilde{a}_{2}, \ldots, \tilde{a}_{p}, \tilde{b}_{1}, \tilde{b}_{2}, \ldots, \tilde{b}_{p}$ are real coefficients, such that $H(j \omega)=|G(j \omega)|^{2}$ and $D_{G}(s)$ is stable (i.e. its roots belong to the open left-half plane).

Lemma 2 Let $f(\cdot)$ be a zero-mean stationary gaussian process whose autocovariance function $h(\cdot)$ satisfies Assumption A1. Consider the system $\mathcal{T}$ defined by ( 6 ) with

$$
\begin{aligned}
A & =\left[\begin{array}{ccccc}
0 & 1 & 0 & \cdots & 0 \\
0 & 0 & 1 & \cdots & 0 \\
\vdots & \vdots & \vdots & \ddots & \vdots \\
0 & 0 & 0 & \cdots & 1 \\
-\tilde{b}_{p} & -\tilde{b}_{p-1} & -\tilde{b}_{p-2} & \cdots & -\tilde{b}_{1}
\end{array}\right], B=\left[\begin{array}{c}
0 \\
0 \\
\vdots \\
0 \\
1
\end{array}\right] \\
C & =\left[\begin{array}{ccccc}
\tilde{a}_{p} & \tilde{a}_{p-1} & \cdots & \tilde{a}_{2} & \tilde{a}_{1}
\end{array}\right] .
\end{aligned}
$$

where the coefficients $\tilde{a}_{i}$ and $\tilde{b}_{i}$ are those of Lemma 1. Let $\frac{d w(t)}{d t}$ be a continuous-time white gaussian noise with unit intensity (or equivalently let the Wiener process $w(\cdot)$ have incremental variance equal to $d t$ ), and $x_{0} \sim N(0, P)$, where $P$ is the unique positive-definite solution of the Lyapunov equation

$$
A P+P A^{\prime}+B B^{\prime}=0 .
$$

Then $\tilde{f}(t)$ has the same statistics as $f(t), \forall t \geq t_{0}$, i.e. $(6)$ is a stochastic state-space realization of $f(\cdot)$.

Example 1 Let $h\left(\left|\xi_{1}-\xi_{2}\right|\right)=\exp \left(-a\left|\xi_{1}-\xi_{2}\right|\right), a>0$, that is the autocovariance function characterizing the Ornstein-Uhlenbeck process. It is easy to show that $H(s)=\frac{2 a}{a^{2}-s^{2}}, G(s)=\frac{\sqrt{2 a}}{s+a}$ and from Lemma 2, $A=-a, B=1, C=\sqrt{2 a}$. Moreover the Lyapunov equation (7) gives $P=\frac{1}{2 a}$.

The next step is to obtain a state-space stochastic realization of the discrete-time process $y_{k}$.

Lemma 3 Let $y_{k}$ be given by (1), where $f(\cdot)$ is a zeromean stationary gaussian process whose autocovariance function $h(\cdot)$ satisfies Assumption A1. Consider the discrete-time time-varying system

$$
\mathcal{W}:\left\{\begin{aligned}
x_{k+1} & =F_{k} x_{k}+G_{k} w_{k} \\
\tilde{y}_{k} & =C x_{k}+\epsilon_{k} \\
x_{0} & \sim N(0, P)
\end{aligned}\right.
$$

where $w_{k} \sim W G N(1)$ (i.e. $w_{k}$ is a discrete-time white Gaussian noise with unit variance), $C$ and $P$ are defined as in Lemma 2, $F_{k}=e^{A\left(t_{k+1}-t_{k}\right)}$, and $G_{k} \in R^{p \times 1}$ is such that

$$
G_{k} G_{k}^{\prime}=\int_{t_{k}}^{t_{k+1}} e^{A\left(t_{k+1}-t\right)} B B^{\prime} e^{A^{\prime}\left(t_{k+1}-t\right)} d t .
$$

Moreover, assume that $w_{k}, \epsilon_{k}, x_{0}$ are mutually independent. Then, the samples $\tilde{y}_{k}$ and $y_{k}, k=1,2, \ldots, n$ have the same statistics, i.e. (8) is a stochastic state space realization of the discrete-time process $y_{k}$.

Now, we can take advantage of the state-space model (8) in order to derive an $O(n)$ algorithm based on Kalman filtering for the computation of the coefficients $\theta_{i}$. For this purpose, a basic property of the Kalman filter is first recalled $[1]$.

Lemma 4 The system

$$
\begin{aligned}
& \mathcal{K}:\left\{\begin{array}{l}
\hat{x}_{k+1}=\left(F_{k}-K_{k} C\right) \hat{x}_{k}+K_{k} \tilde{y}_{k} \\
\nu_{k}=\left(R_{k}^{e}\right)^{-\frac{1}{2}}\left(\tilde{y}_{k}-C \hat{x}_{k}\right) \\
x_{1}=0
\end{array}\right. \\
& R_{k}^{e}=C \Sigma_{k} C^{\prime}+\sigma^{2} \\
& K_{k}=F_{k} \Sigma_{k} C^{\prime}\left[C \Sigma_{k} C^{\prime}+\sigma^{2}\right]^{-1} \\
& \Sigma_{k+1}=F_{k} \Sigma_{k} F_{k}^{\prime}+ \\
&-F_{k} \Sigma_{k} C^{\prime}\left[C \Sigma_{k} C^{\prime}+\sigma^{2}\right]^{-1} C \Sigma_{k} F_{k}^{\prime}+G_{k} G_{k}^{\prime} \\
& \Sigma_{1}=F_{0} P F_{0}^{\prime}+G_{0} G_{0}^{\prime},
\end{aligned}
$$


is a "whitening filter" for the sequence $\tilde{y}_{k}, k=$ $1,2, \ldots, n$, defined in Lemma 3, in the sense that $\nu_{k} \sim$ $W G N(1)$.

We are now in a position to state the main result.

Theorem 5 The weights $\theta_{2}, i=1, \ldots, n$, in (4), (5), can be computed by means of the following algorithm

$$
\begin{aligned}
\hat{x}_{k+1} & =\left(F_{k}-K_{k} C\right) \hat{x}_{k}+K_{k} y_{k}, \hat{x}_{1}=0 \\
\nu_{k} & =\left(R_{k}^{e}\right)^{-\frac{1}{2}}\left(y_{k}-C \hat{x}_{k}\right), k=1,2, \ldots, n \\
\xi_{j-1} & =\left(F_{j}-K_{j} C\right)^{\prime} \xi_{j}-C^{\prime}\left(R_{j}^{e}\right)^{-\frac{1}{2}} \nu_{j}, \xi_{n}=0(10) \\
\theta_{j} & =K_{j}^{\prime} \xi_{j}+\left(R_{j}^{e}\right)^{-\frac{1}{2}} \nu_{j}, j=n, n-1, \ldots, 1 .
\end{aligned}
$$

where $K_{k}$ and $R_{k}^{e}$ are defined as in Lemma 4 .

Proof: : Omitted.

Summarizing the results of this section, the proposed algorithm consists of the following steps:

1. Compute the bilateral Laplace transform $H(s)=$ $\mathcal{L}(h)(s)$

2. Use the constructive procedure outlined in the proof of Lemma 3 to compute the polynomials $N_{G}(s), D_{G}(s)$;

3. Construct matrices $A, B, C$, according to Lemma 2 ;

4. Compute the $F_{k}$ matrices as $F_{k}=e^{A\left(t_{k+1}-t_{k}\right)}$, $k=1,2, \ldots, n$

5. Compute the $P$ matrix by solving equation (7) (this requires $O\left(p^{3}\right)$ operations [9]);

6. For $k=1,2, \ldots, n$ compute the matrices

$$
Q_{k}=G_{k} G_{k}^{\prime}=\int_{0}^{t_{k+1}-t_{k}} e^{A \tau} B B^{\prime} e^{A^{\prime} \tau} d \tau
$$

This may be done by the means of the following procedure [14]

$$
\begin{gathered}
\ddot{F} \doteq\left[\begin{array}{ll}
-A & B B^{\prime} \\
0 & A
\end{array}\right],\left[\begin{array}{ll}
\Phi_{1} & \Psi \\
0 & \Phi_{2}
\end{array}\right] \doteq e^{\bar{F}\left(t_{k+1}-t_{k}\right)}, \\
\Phi_{1} \in \mathbb{R}^{p \times p}, Q_{k}=\Phi_{2}^{\prime} \Psi
\end{gathered}
$$

7. Compute the $\theta_{k}$ coefficients using the algorithm of Theorem 5 .
The computational cost of steps 4 and 6 scales as $O\left(p^{3} n\right)$, where the factor $p^{3}$ arises from the calculation of the exponential matrices [8]. However, we stress that $p$ is fixed (and usually $p \ll n$ ) so that the complexity of these steps scales in a linear way with the size of the dataset. Moreover, it is easily seen by inspection that the Kalman filter calculations involved in Theorem 5 take $O\left(p^{3} n\right)$ operations [1]. Hence, the overall complexity of the proposed algorithm is linear with respect to the number of the collected datapoints.

\section{Further generalizations}

A careful examination of Lemmas 3 and 4 and Theorem 5 reveals that their proofs are independent of the structure of the matrices $A, B, C$. In fact, their statements still hold true provided that the Bayesian prior is assigned in state-space (i.e. Markovian) form: more precisely one has to assume that $f(t)=\tilde{f}(t)$ with $\tilde{f}(t)$ defined by (6) where $\frac{d w(t)}{d t}$ is a Gaussian white noise with unitary intensity and $x_{0} \sim N(0, P)$, where $P>0$ is an arbitrary matrix (it need not satisfy the Lyapunov equation (7)). Note that, when $P$ does not satisfy (7), the process $\tilde{f}(t)$, is no more stationary. An interesting case is given by the following choice of the system $\mathcal{T}$ :

$$
\begin{aligned}
A & =\left[\begin{array}{cccc}
0 & 0 & \cdots & 0 \\
1 & 0 & \cdots & 0 \\
\vdots & \ddots & \ddots & \vdots \\
0 & \cdots & 1 & 0
\end{array}\right], B=\left[\begin{array}{c}
1 \\
0 \\
\vdots \\
0
\end{array}\right] \\
C & =\left[\begin{array}{llll}
0 & \cdots & 0 & 1
\end{array}\right],
\end{aligned}
$$

where $A \in \mathbb{R}^{p \times p}$. If $x_{0}=N(0,0)$, it is easy to recognize that $\tilde{f}(t)$ is a $(p-1)$ fold integrated Wiener process and the associated Bayes estimate turns out to be a $p$-th order regression spline with zero boundary conditions [15]. Then, steps 4,6 and 7 of the algorithm presented in the previous section provide an $O(n)$ procedure for the computation of the spline weights.

Unfortunately, it is not possible to extend the $O(n)$ algorithm to the general multidimensional case $(d>$ 1). However, as explained below, it is possible to take advantage of the proposed algorithm when computing the weights of a particular class of RNs, namely additive RNs.

Assume that $f(\cdot): \mathbb{R}^{d} \rightarrow \mathbb{R}$ takes the form

$$
f(t)=\sum_{\mu=1}^{d} f_{\mu}\left(t^{\mu}\right), \quad t=\left[\begin{array}{llll}
t^{1} & t^{2} & \cdots & t^{d}
\end{array}\right]^{\prime}
$$

where $f_{\mu}(\cdot): \mathbb{R} \rightarrow \mathbb{R}$ represent the additive components. Assume that the Bayesian paradigm is adopted. If such functions are modeled as independent zero-mean Gaussian processes with autocovariances $C_{\mu}\left(\xi_{1}^{\mu}, \xi_{2}^{\mu}\right), \mu=1,2, \ldots, d$, then $f(\cdot)$ is a zero- 
mean Gaussian process with autocovariance

$$
C\left(\xi_{1}, \xi_{2}\right)=\sum_{\mu=1}^{d} C_{\mu}\left(\xi_{1}^{\mu}, \xi_{2}^{\mu}\right)
$$

Obviously, the weights $\bar{\theta}$ of the associated additive RN can be computed from the training set $\mathcal{D}_{n}$ via (3). However, under mild assumptions [10], the Bayes estimate can also be computed as

$$
\hat{f}(t)=\sum_{\mu=1}^{d} \hat{f}_{\mu}\left(t^{\mu}\right)
$$

where the functions $\hat{f}_{\mu}$ are obtained via the so called backfitting algorithm. This procedure amounts to the recursive estimation of the monodimensional functions $\hat{f}_{\mu}$ and it converges to the correct estimate under mild assumptions [10]. Moreover, at each iteration $i$, the functions $\hat{f}_{\mu}^{i}$ can be computed as RNs that use a modified set of samples $y_{i}$. Then, if $C_{\mu}\left(\xi_{1}^{\mu}, \xi_{2}^{\mu}\right)$ satisfy Assumption $\mathrm{A} 1$, the components $\hat{f}_{\mu}^{i}$ can be computed by using the algorithm presented in Section 3 . In this case, if the backfitting procedure stops after $\bar{n}<n^{2}$ iterations, the overall computational complexity is $O(\bar{n} n)$ that is less than the $O\left(n^{3}\right)$ operations required by the direct solution of the linear system (3).

\section{Concluding remarks}

It has been shown that for a fairly large class of monodimensional RNs, the weights can be computed in $O(n)$ operations via a Kalman filtering algorithm. Unfortunately, the algorithm cannot be extended to the general multidimensional case. A notable exception, however, is given by additive RNs: if their weights are computed using the backfitting procedure, one obtains a sequence of monodimensional problems that can be solved in $O(n)$ operations via Kalman filtering.

\section{References}

[1] B.D.O. Anderson and J.B. Moore. Optimal Filtering. Prentice-Hall, Englewood Cliffs, New Jersey, 1979.

[2] C.F. Ansley and R. Kohn. Estimation, filtering, and smoothing in state space models with incompletely specified initial conditions. Ann. Statist., 13(4):1286$1316,1985$.

[3] V. Corradi and H. White. Regularized neural networks: some convergence rate results. Neural Computation, 7:1225-1244, 1995.

[4] G. De Nicolao and G. Ferrari-Trecate. Consistent identification of NARX models via regularization networks. IEEE Trans. Autom. Contr., 44(11):2045-2049, 1999.
[5] A. Gentilini, M. Rossoni-Gerosa, C.W. Frei, and M. Morari. Pharmacokinetic pharmacodynamic modeling of bispectral index with isoflurane for closed loop control of depth of anaesthesia. Proc. World Congress of Medical Physics and Biomedical Engineer, 2000.

[6] F. Girosi. An equivalence between sparse approximation and support vector machines. Neural Computation, 10:1455-1480, 1998.

[7] F. Girosi, M. Jones, and T. Poggio. Regularization theory and neural networks architectures. Neural Computation, 7:219-269, 1995.

[8] G.H. Golub and C.F. Van Loan. Matrix computations. The Johns Hopkins University Press, Baltimore, Maryland, 1984.

[9] S.J. Hammarling. Numerical solution of the stable, non-negative definite Lyapunov equation. IMA J. of Numerical Analysis, 2:303-323, 1982.

[10] T.J. Hastie and R.J. Tibshirani. Generalized additive models. Chapman and Hall, London, 1990.

[11] A. Papoulis. Probability, random variables and stochastic processes. McGraw-Hill, New York, 1991.

[12] T. Poggio and F. Girosi. Networks for approximation and learning. IEEE Proc., 78:1481-1497, 1990.

[13] W. Rudin. Real and Complex Analysis. McGrawHill, 1987.

[14] C.F. Van Loan. Computing integrals involving the matrix exponential. IEEE Trans. Autom. Control, 23:395-404, 1978.

[15] G. Wahba. Spline Models for Observational Data. SIAM, Philadelphia, 1990.

[16] H.L. Weinert, R.H. Byrd, and G.S. Sidhu. A stochastic framework for recursive computation of spline functions: Part ii, smoothing splines. J. Optim. Th. and Appl., 30(2):255-268, 1980.

[17] P. Whittle. Prediction and regulation by linear least-square methods. English Universities Press, 1963.

[18] C.K.J. Williams and C.E. Rasmussen. Gaussian processes for regression. In D.S. Touretzky, M.C. Mozer, and M.E. Hasselmo, editors, Advances in Nueral Information Processing Systems, 8. MIT Press, 1996. 